FORMATION Formation emploi

Revue française de sciences sociales

116 | octobre-décembre 2011

Pêle-mêle

\title{
Les marchés du travail et de la formation :entre singularités et proximités
}

Le cas du collaborateur social de cabinet d'expertise comptable et d'une licence professionnelle

Labour markets and training: specificity vs, proximity: The case of the lay employee at an accounting firm and a vocational degree

Arbeits- und Ausbildungsmärkte:zwischen Besonderheiten und Proximitäten

Der Fall des „Collaborateur social de cabinet d'expertise comptable“ (Assistent in einer Buchprüfungsgesellschaft) und einer „Licence professionnelle“

(bachelorähnlicher Abschluss)

Los mercados de trabajo y formación : entre singularidades y proximidades

El caso del colaborador social de un estudio contable y de una licenciatura

profesional

\section{Anne Moysan-Louazel}

\section{OpenEdition}

Journals

Édition électronique

URL : http://journals.openedition.org/formationemploi/3448

DOI : 10.4000/formationemploi.3448

ISSN : 2107-0946

Éditeur

La Documentation française

Édition imprimée

Date de publication : 30 décembre 2011

Pagination : 19-34

ISSN : 0759-6340

Référence électronique

Anne Moysan-Louazel, "Les marchés du travail et de la formation :entre singularités et proximités », Formation emploi [En ligne], 116 | octobre-décembre 2011, mis en ligne le 03 février 2012, consulté le 30 octobre 2020. URL : http://journals.openedition.org/formationemploi/3448 ; DOI : https://doi.org/ 10.4000/formationemploi.3448 


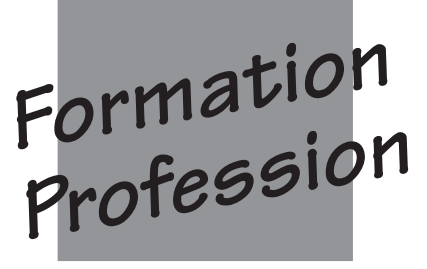

\section{Les marchés du travail et de la formation : entre singularités et proximités}

\section{Le cas du collaborateur social de cabinet d'expertise comptable et d'une licence professionnelle}

Anne Moysan-Louazel*

La licence professionnelle est un diplôme fondé sur des partenariats entre université et acteurs économiques. On passe alors, non sans remises en question, de partenariats locaux à une tentative de régulation nationale.

Le marché de la formation est-il vraiment un marché « comme les autres »? La question n'est pas nouvelle (D’Iribarne, 2003). Elle mérite cependant d'être à nouveau posée dans le contexte actuel. L'État et les grandes institutions de niveau national confient de plus en plus à des acteurs territoriaux le soin de s'ajuster entre eux et de produire les normes propres à leurs contextes locaux. Cela a été particulièrement le cas lors de la création des licences professionnelles. La diversité de ces « arrangements institutionnels $»^{1}$ a été notamment soulignée par M. Bel (2005) qui parle de coordinations « situées » pour rendre compte de cette variété. Conformément aux textes officiels, l'éla-

1 Gautié et alii. (2005) parlent aussi de «mode d'organisation des transactions caractéristiques de certains marchés ».
* Anne Moysan-Louazel est économiste, maître de conférences à l'université de Rennes 1, CREM Centre de recherche en économie et management UMR CNRS 6211. Elle a récemment publié : "Les professions libérales réglementées, le marché et la concurrence. Le cas des experts-comptables et des avocats », Comptabilité, Contrôle, Audit, septembre 2011 . "Le Fongecif, territoire d'équité ou d'égalité pour la formation continue ? », avec S. Ghaffari, A. Giffard, C. Guégnard, G. Podevin, Travail et Emploi, $n^{\circ} 116$, décembre 2008. "Une nouvelle dynamique des marchés du travail réglementés, à l'aune de trois professions », avec Gérard Podevin, Formation Emploi revue française de sciences sociale, $n^{\circ} 102$, pp. 5-19, avril-juin, 2008. 
boration de ces licences nécessite une mise en relation entre les organismes de formation et les milieux professionnels. Il s'agit d'évaluer les besoins, d'objectiver et étalonner les compétences mobilisées dans l'exercice professionnel et participer au déroulement de la formation. La participation des acteurs économiques est certes indispensable à l'ouverture de ces formations ; cependant, les motifs de l'investissement de ces acteurs dans la construction et la mise en œuvre de la formation sont divers. La compréhension des jeux d'acteurs sur ce marché et des dispositifs de coordination auxquels ils ont recours est donc cruciale.

Le rôle de la proximité dans l'efficacité de l'appariement entre offre et demande sur les marchés locaux du travail est connu (Bouba-Olga, Bourdu, 2009). On sait aussi, depuis les travaux de L. Karpik (2007), que la « singularité » de certains marchés nécessite des dispositifs de coordination particuliers. Nous proposons ici de mettre en évidence l'intérêt de cette double perspective théorique pour appréhender la dynamique des dispositifs de coordination sur un marché du travail qui, en l'absence de compétences préexistantes, se « ferme » pour prendre en charge la formation des candidats. Ce marché du travail se caractérise alors par une proximité importante entre le système de production et le système de formation. À cet effet, nous nous intéresserons plus particulièrement à la création et à l'évolution récente de la licence professionnelle «Assistant paie et administration du personnel ». Notre article met en évidence l'évolution, dans le temps, des motifs qui ont conduit à l'ouverture de ce diplôme, ainsi que du modèle de coordination et du glissement des formes de proximité.

Cette licence est issue d'un partenariat entre un Conseil régional, l'Ordre des experts-comptables et un IUT (Institut universitaire de technologie). Notre participation à sa création constitue un terrain d'observation et d'analyse particulièrement riche pendant une période longue de huit années (encadré 1). Que nous apprennent les observations réalisées sur les comportements des acteurs et des intermédiaires du marché du travail et de la formation et les formes de proximité mobilisées ? Quel est le rôle joué par le dispositif de formation dans la mise en forme du marché du travail du collaborateur social ?
La première partie de cet article est consacrée à la présentation du métier de collaborateur social et aux motifs et au processus qui ont conduit à l'ouverture de la licence. Nous nous attachons à mettre à jour les principaux moments de la construction puis de l'évolution récente de la licence. Dans une seconde partie, les concepts de l'école de la proximité et de l'économie des singularités sont mobilisés pour analyser la coordination entre les acteurs des marchés du travail et de la formation et interpréter les évolutions récentes.

\section{UNE LICENCE POUR PROFESSIONNALISER LE MÉTIER DE COLLABORATEUR SOCIAL}

Les besoins en recrutement et en formation des cabinets d'expertise comptable se sont modifiés sur la période récente, sous l'effet de changements techniques, organisationnels et réglementaires. Ces évolutions les ont conduits à arbitrer entre formation interne, c'est-à-dire formation continue ou « sur le tas », et formation externe, via le recrutement de diplômés sur le marché du travail.

Avant d'analyser les ressorts et les modalités de cet arbitrage, et donc les motifs qui ont présidé à la création de la licence, nous présentons le métier encore peu connu de collaborateur social en cabinet d'expertise comptable.

\section{Le collaborateur social : un métier émergent}

La profession comptable est actuellement confrontée à de nombreux défis. Bien que réglementée, elle évolue dans un environnement de plus en plus concurrentiel : volonté des autorités de déréglementer la profession pour éliminer les obstacles et ouvrir le marché européen de services professionnels à la concurrence ${ }^{2}$,

2 De fortes pressions émanant d'acteurs publics et privés européens et internationaux pèsent sur l'État pour démanteler ces marchés protégés. Le bien-fondé des réglementations professionnelles procurant aux professions libérales des protections de dimension nationale à l'aide d'exigences de qualifications et de diplômes ainsi que celles relatives aux tâches réservées est en discussion. 


\section{Encadré 1}

\section{Méthodologie}

Cet article repose sur l'expérience de l'auteur, à l'origine de la création de la licence professionnelle "Assistant paie et administration du personnel ", et toujours en charge de la responsabilité de cette formation à l'université de Rennes 1. Responsabilité qui implique l'accompagnement des cabinets d'expertise comptable dans leur recrutement de stagiaires puis de diplômés ainsi que l'accompagnement de collaborateurs déjà en poste dans leur démarche de VAE (validation des acquis de l'expérience) et leur projet professionnel.

II s'appuie sur des données qualitatives issues de plusieurs sources:

- Des témoignages d'acteurs du milieu professionnel et de la formation recueillis de manière non systématique et formalisés entre 2002 et 2010 . Les entretiens ont été réalisés auprès d'experts-comptables, de représentants de l'Ordre et de l'OPCA (Organisme paritaire collecteur agréé) de la branche. De nombreux entretiens ont été réalisés auprès de responsables de service social en cabinet d'expertise comptable dans le cadre de visite de stage d'étudiants en licence et en amont de leur placement en contrat de professionnalisation.

- La réalisation d'une monographie de cabinet d'expertise comptable en 2008-2009, dans le cadre de l'enquête sur les professions intermédiaires en entreprise (EPIE) du Cereq. Celle-ci visait à cerner les pratiques de professionnalisation à l'œuvre dans les entreprises pour ces catégories /administratifs, commerciaux, techniciens, maîtrise). Une trentaine d'entreprises ont été enquêtées entre 2008 et 2010. Dans le cadre de cette enquête, sept entretiens semi-directifs ont été réalisés entre novembre 2008 et février 2009 auprès de dirigeants et de collaborateurs du cabinet d'expertise comptable.

- Des documents et textes :

- Des textes relatifs à la branche (convention collective, accord formation, Opca...)

- Des revues professionnelles: SIC, le magazine de l'Ordre des experts-comptables; la profession comptable; les revues éditées par les conseils régionaux de l'Ordre des experts-comptables, etc.

- Des documents internes à l'IUT (Institut universitaire de technologie) de Rennes relatifs à la gestion de la licence : comptes-rendus de comité de perfectionnement de la licence, du comité de gestion du service de formation continue de l'IUT, évaluations de la formation par les entreprises et les cabinets, enquêtes annuelles d'insertion des diplômés, etc.

arrivée de nouveaux intervenants ${ }^{3}$ qui entrent en concurrence directe avec les cabinets d'expertise comptable sur le marché non réglementé des services de conseil fiscal, social et financier. Les enjeux sont toutefois variables selon la taille des cabinets. La diversification des prestations n'est pas perçue comme un avantage concurrentiel par tous. Si les plus petits cabinets réalisent encore l'essentiel de leur activité dans le cadre des prestations réglementées, c'est-àdire les prestations comptables traditionnelles, les plus importants d'entre eux tirent une partie importante de leur chiffre d'affaires de prestations non récur-

\footnotetext{
3 Cabinets de conseil ; avocats ; banques ; fiscalistes ; éditeurs de
} logiciels de comptabilité et de paie. rentes et non réglementées ${ }^{4}$. Le développement de réseaux de cabinets indépendants et de cabinets pluridisciplinaires, la création de filiales et de divisions sectorielles permettent de mutualiser le coût de ces services professionnels; ceux-ci sont intégrés dans un ensemble de prestations globales, débordant ainsi de leur domaine de compétence légale.

Ces transformations les conduisent à rechercher de nouvelles compétences et à « construire » de nouveaux métiers. Le poids des charges sociales, une réglementation sociale de plus en plus complexe et difficile à maîtriser et en constante évolution, conjugués à la

\footnotetext{
4 Citons par exemple : conseil en droit social ; paie ; optimisation de la rémunération du dirigeant, simulation de l'impact d'une nouvelle embauche; conseils en fusion-acquisition, etc.
} 
difficulté à identifier et instrumentaliser les leviers de la motivation du personnel sont des problématiques majeures pour les chefs d'entreprise. Ces questions peuvent faire l'objet d'une stratégie de diversification pour certains cabinets dans le cadre d'une externalisation des missions « social et $\mathrm{RH}$ » plus ou moins étendue (Moysan-Louazel, Merel, 2008). Nos observations de terrain ont montré que les cabinets d'expertise comptable peuvent être amenés à assurer, pour le compte de leurs clients, les tâches suivantes : gestion administrative du personnel (contrat de travail, paie, déclarations sociales, régimes de retraite et de prévoyance, maladies, accidents du travail,...) ; recrutement (aide au choix du contrat, contrats aidés, définition de poste,...) ; gestion de l'activité (temps de travail, absences, maladie,...) ; politique de rémunération et masse salariale (intéressement, participation,...) ; audit social, de conformité ; gestion de l'emploi, des carrières, des compétences, de la formation (DIF - droit individuel à la formation -, Plan formation,...) ; relations sociales (négociation collective, dialogue social, organisation des élections des RP,...) ; aide au choix de logiciel RH et paie, formation,...).

La profession comptable libérale prend progressivement conscience des potentialités de ce marché mais aussi des impératifs de professionnalisation des collaborateurs que cela implique. "Si on veut assurer un service de qualité, très sécurisé et professionnalisé, il nous faut nécessairement des intervenants spécialisés » (Jean-Pierre Alix, ancien Président du Conseil supérieur de l'Ordre des experts-comptables, 2006).

L'activité « social et RH », dans sa version de base qui consiste à réaliser la paie des clients, n'est pas nouvelle dans les cabinets. Elle a d'abord été confiée aux collaborateurs comptables et s'est progressivement ajoutée à la mission comptable. Cette origine explique qu'elle soit méconnue à l'heure actuelle et la confusion qui persiste entre le métier de collaborateur social et celui de collaborateur comptable. Cette confusion est entretenue par l'hétérogénéité de leur travail et de leur position au sein des cabinets. Le métier de collaborateur social est un métier émergent dans les cabinets d'expertise comptable. La création d'un poste de collaborateur social exige un volume de prestations suffisant. Le profil de poste et le type de responsabilités qui lui sont confiées restent donc encore à l'heure actuelle assez différents d'un cabinet à un autre. Il est généralement attendu du collaborateur social qu'il ait des compétences juridiques et techniques en droit social et paie ainsi qu'en gestion des ressources humaines (encadré 2).

Quand l'activité sociale existe dans le cabinet, elle représente un enjeu stratégique à plusieurs niveaux : pour optimiser et fiabiliser la production de la paie tout en effectuant des gains de productivité ; pour développer et vendre de nouvelles missions de conseil auprès des clients existants et de nouveaux clients. Pourtant, le social reste une matière souvent peu appréciée des collaborateurs en raison de ses aspects complexes, évolutifs et relationnels. De leur côté, les dirigeants des cabinets mettent en avant les nombreux risques d'erreurs et la faible rentabilité générée par ce type de service en raison du coût de production du bulletin de paie, du volume d'activité et de la difficulté à facturer une prestation distincte longtemps " offerte » aux clients dans le cadre d'une prestation comptable. La gestion des compétences et de la professionnalisation des collaborateurs devient une préoccupation pour beaucoup de cabinets peu accoutumés au management des ressources humaines (RH). Les enjeux sont nombreux, qu'il s'agisse de spécialiser les collaborateurs et les départements, de diversifier les profils ou encore d'organiser des parcours de mobilités internes pour fidéliser et motiver les collaborateurs. Et ce, dans un contexte où les cabinets souffrent d'un problème d'attractivité (particulièrement au sein de la jeune génération) en raison des faibles niveaux de rémunération et des rythmes de travail très soutenus à certaines périodes de l'année.

La profession comptable libérale, dans son ensemble, peine à renouveler son image et à communiquer sur l'évolution du contenu de ses activités (réglementées et non réglementées) et des métiers offerts en cabinet. "L'expérience Cabinet a très bonne presse auprès des entreprises » (Responsable Agence d'Intérim) mais au-delà de cette image "d'école de formation " assurant un apprentissage de la technicité, les perspectives de carrière sont réduites. Les cabinets d'expertise comptable ne sont pas en situation, pour la grande majorité d'entre eux ${ }^{5}$, de créer des marchés internes

\footnotetext{
5 À la fin décembre 2005, on dénombrait 12300 cabinets ayant un effectif de 0 à 50 salariés contre 130 environ ayant un effectif supérieur à 50 salariés (CSOEC, 2006).
} 


\section{Encadré 2}

\section{Compétences ou capacités attestées du collaborateur social}

Les compétences requises pour exercer cette fonction sont techniques et juridiques. Les qualités managériales et relationnelles sont également de plus en plus importantes.

\section{PAIE}

Réaliser en autonomie complète la gestion de la paie à l'aide de logiciels professionnels ; Réaliser l'ensemble des déclarations sociales et fiscales de paie à l'aide de logiciels professionnels; Disposer de bonnes connaissances en droit du travail et droit social pour être capable de s'assurer de la conformité des activités et exercer une activité de conseil ;

Analyser les lois, les décrets et les conventions collectives et leurs impacts sur la gestion de la paie.

\section{DROIT}

Connaître la diversité des contrats de travail et leurs spécificités ;

Connaître le statut juridique, social et fiscal des dirigeants d'entreprise ;

Identifier et prévenir le risque contentieux en matière sociale ;

Développer le sens de la veille sociale, juridique et économique ;

Etre un interlocuteur reconnu en matière de législation sociale et assurer les relations avec les organismes sociaux et fiscaux ;

Participer à la mise en place des institutions représentatives du personnel ;

Connaître les règles applicables à chaque niveau de négociation collective ;

Maitriser les connaissances de base de l'intéressement et de la participation pour accompagner les dirigeants d'entreprise dans la mise en place d'accords.

\section{GESTION DES RESSOURCES HUMAINES}

Analyser les impacts financiers de la politique de rémunération ;

Appréhender les outils de mesure de la performance sociale ;

Élaborer des tableaux de bord sociaux permettant de suivre l'activité du personnel : absentéisme, rémunérations, congés, accidents du travail... et analyser les données recueillies.

Réaliser un audit social ;

Participer à l'élaboration du plan de formation de l'entreprise ;

Participer à la gestion et l'anticipation des risques professionnels ;

Participer à la mise en place d'une gestion des compétences et des parcours professionnels ;

Participer au recrutement.

Source : Extrait fiche RNCP (Répertoire national des certifications professionnelles) de la licence professionnelle « Assistant paie et administration du personnel » (2010).

caractérisés par l'existence de chaîne de mobilité. Les filières de mobilité sont courtes. Le passage par un poste de « Paie » est perçu comme une «porte d'entrée » dans la fonction RH en entreprise. À ce problème d'image s'ajoute un « déficit de langage » de la part des cabinets qui rencontrent parfois des difficultés à faire connaître leurs besoins. Les métiers liés à l'activité « Paie et RH » des cabinets sont mal recensés et mal identifiés par les jeunes et les salariés.
Le poste de collaborateur en cabinet reste associé à des tâches purement comptables. Cela se traduit par des difficultés à recruter ${ }^{6}$. Dès lors, la pénurie exprimée ne

\footnotetext{
6 De nombreux témoignages attestent d'une apparente pénurie sur la qualification de collaborateur social. Si un sondage réalisé par l'IFEC (Institut français des experts-comptables et des commissaires aux comptes, ) du 30 avril au 6 mai 2007, révèle que $83 \%$ des votants estiment rencontrer des difficultés de recrutement des collaborateurs comptables, aucune enquête statistique mettant en évidence le rapport entre les offres et les demandes d'emploi enregistrées ne permet cependant de confirmer la pénurie sur le
} 
masque-t-elle pas, en réalité, des difficultés d'ajustement sur le marché du travail et une émergence et/ou formalisation de la fonction RH encore embryonnaire au sein de nombreux cabinets d'expertise comptable?

Devant les difficultés rencontrées pour développer, au sein de leurs cabinets - dans une logique de marchés internes du travail, c'est-à-dire par l'alimentation des emplois par la voie de la mobilité interne - les compétences requises pour exercer le métier de collaborateur social, certains représentants de la profession comptable libérale choisissent d'inscrire la professionnalisation et la GRH de leurs collaborateurs dans une logique de marchés professionnels en créant, avec un IUT, un diplôme professionnel. Selon un responsable de service social d'un cabinet d'expertise comptable "la matière paie et sociale s'est complexifiée. Le métier de collaborateur social devient un métier d'expertise. On assiste à une professionnalisation $d u$ métier. Avant, on apprenait sur le tas. Désormais, il est nécessaire d'avoir une vraie formation. Aujourd'hui, on recrute à un niveau plus élevé (bac $+2-3)$ ».

Pourtant, d'autres professionnels soulignent l'importance de l'exercice de la pratique professionnelle dans la durée pour devenir un «vrai » professionnel. Selon eux, seules la pratique et la confrontation progressive puis régulière avec les clients permettent l'acquisition des compétences relationnelles et comportementales. Sur le plan des compétences techniques, la connaissance et l'application de la réglementation et de son évolution constante excluent le recours à la formation initiale comme seule modalité d'acquisition. L'apprentissage sur le tas est considéré comme un complément indispensable aux connaissances théoriques pour faire face aux situations professionnelles réelles et à une relation avec les clients parfois tendue.

La formation initiale constitue certes une étape dans la professionnalisation des collaborateurs ; cependant, elle est surtout sollicitée lors de la phase d'intégration et pour faciliter le recrutement en constituant un vivier de candidats potentiels.

Les modalités d'acquisition de compétences semblent donc intimement liées à l'exercice de l'activité « entre

métier de collaborateur social, d'autant qu'aucune augmentation générale de salaires n'a été observée. pairs ». Elles rendent difficiles la formalisation mais aussi l'appréciation de la qualité de la formation en amont du recrutement. C'est la raison pour laquelle, loin de pratiquer une " cueillette des qualifications » (Chassard et Kerbourc'h, 2007), c'est-à-dire de demander à l'IUT de leur fournir une main-d'œuvre immédiatement utilisable, les cabinets vont accepter de co-construire l'offre de formation, reconnaissant qu'ils sont à la fois utilisateurs et producteurs de qualifications.

Au-delà de leur rôle de facilitateur de l'appariement entre une offre et une demande déjà constituée, les différents acteurs vont contribuer à structurer le marché en participant à l'expression de l'offre et de la demande de formation. La licence prépare l'insertion dans l'emploi. Elle favorise en outre une évaluation du futur collaborateur proche des contextes professionnels et un appariement entre lui et l'employeur en deux temps (à l'entrée en formation et pendant les périodes de stage). En ce sens, la licence est une construction institutionnelle qui, par la confiance qu'elle a créée entre les acteurs, va permettre de produire de l'information nécessaire au recrutement.

\section{Genèse et évolution de la licence}

À l'image de toutes les licences professionnelles (Maillard, Veneau, Grandgérard, 2004), celle que nous étudions ici a été construite en référence à un métier et donc à l'acquisition des compétences nécessaires à son exercice. Sa " singularité » tient en partie au caractère émergent du métier qui rend encore plus indispensable la coordination avec les professionnels pour acquérir l'information sur les compétences à transmettre. Les différents acteurs du marché (université, professionnels, enseignants, étudiants) rencontraient un problème d'informations sur le contenu du métier et les compétences à construire.

L'ouverture de la licence a été précédée d'une première étape dans le processus de professionnalisation du métier de collaborateur social. Créé en 1996, le Comité social de l'Ordre a mise en place une formation certifiante de douze jours, créée en Bretagne en 1999, qui s'est ensuite étendue à d'autres régions. Mais rapidement la mobilité interne des collaborateurs 
comptables vers des postes de collaborateur social ne suffit plus. En Bretagne, les experts comptables vont alors se tourner vers l'IUT pour constituer un vivier de jeunes collaborateurs en social. Ils vont être localement présents tout au long du processus de création de la licence, puis associés dans sa mise en œuvre : participation au jury de sélection des candidats, aux jurys d'examen, aux jurys de Validation des acquis de l'expérience (VAE), aux enseignements et au comité de perfectionnement. La capacité d'organisation collective de la profession comptable à s'investir dans la construction de la formation va jouer un rôle essentiel au niveau local à travers le Conseil régional de l'Ordre des experts-comptables de Bretagne (CROECB $)^{7}$. Dès 2002, ce dernier s'engage dans un partenariat avec l'IUT de Rennes pour créer un diplôme d'université (DU) qui se transforme en licence en 2004. Soulignons que les relations entre l'IUT et les professionnels préexistaient à la création de la licence. Proposant aux étudiants de deuxième année une orientation vers l'option Finance-comptabilité ou ressources humaines, le département de gestion (GEA - gestion des entreprises et des administrations) de l'IUT avait déjà l'expérience de formations conçues en référence aux métiers de la comptabilité et de la gestion des ressources humaines. Le réseau de relations avec les cabinets d'expertise comptable permettait d'offrir des lieux de stages et de recruter des professionnels sous statut de vacataire pour participer aux enseignements.

La licence prépare non seulement au métier de collaborateur en charge de la paie et de la gestion sociale dans les cabinets d'expertise comptable, mais aussi au métier d'assistant RH pour les PME. Rapidement, la décision a en effet été prise, par la responsable de formation, et avec l'accord des partenaires du Conseil

\footnotetext{
7 Rappelons que les cabinets d'expertise comptable sont des entreprises un peu particulières. Elles évoluent en effet dans un environnement réglementaire et délivrant des prestations normées. La profession comptable est réglementée et dispose d'une institution ordinale. Cette institution, dirigée par des membres de la profession élus par leurs pairs, veille au respect des règles de déontologie. Elle est chargée de l'organisation de la formation initiale et continue de ses membres ainsi que du contrôle des modalités d'accès à la profession et de la régulation démographique. Le partage des rôles attribue à l'Ordre la régulation de la profession et la gestion des relations avec la tutelle ministérielle, tandis que les deux syndicats professionnels négocient la convention collective et défendent la profession. Dans la réalité, les syndicats sont peu représentés dans la profession et «l'acteur principal sur le marché du travail, c'est l'Ordre» (Expert comptable).
}

régional de l'Ordre des experts comptables, d'ouvrir la licence aux entreprises autres que les cabinets d'expertise comptable en leur donnant la possibilité de recruter un stagiaire. Si l'objectif consistait à satisfaire la demande des PME, il s'agissait aussi d'éviter l'enfermement des diplômés au sein des cabinets d'expertise comptable. Les compétences acquises par le stagiaire permettent en effet une mobilité inter sectorielles dans une logique de marché professionnel.

En 2007, le partenariat avec la profession s'est renforcé autour du dispositif de VAE et grâce à la signature d'une convention entre le Conseil régional de l'Ordre des experts comptables de Bretagne (CROECB) et l'université de Rennes 1. Au moment de la création de la licence, un accord tacite avait réservé la formation initiale des futurs collaborateurs à l'IUT et la formation continue des collaborateurs déjà en poste à l'organisme de formation du Conseil régional de l'Ordre (Institut supérieur de formation aux études comptables (ISFEC)). Quelles ont été les motivations des représentants de la profession lors de la signature de la convention? D'une part, ils ont considéré que l'accès au diplôme de la licence pouvait constituer un puissant levier de GRH pour fidéliser les collaborateurs déjà en poste et activer le marché interne. D'autre part, la construction d'un « parcours» combinant les deux modules de formation du « cycle social $»^{8}$ de l'ISFEC, la VAE et une reprise d'études en licence en cas de validation partielle permettait de réduire les coûts de formation mais aussi d'adosser le cursus de l'ISFEC à un diplôme national valorisé par les collaborateurs. Le réseau d'acteurs déjà constitué autour de la licence a permis de construire ce dispositif spécifique combinant différentes ressources.

Aujourd'hui, l'hétérogénéité des missions sociales effectuées en cabinet (en termes de CA et d'effectifs) et des fiches de poste et profils de poste du collaborateur social reste importante. Le lien distendu entre le métier et la formation montre que l'on n'est pas dans une logique de métier ou de " marchés professionnels ».

\footnotetext{
8 Il s'agit des modules de formation cycle 1 et 2 (« Devenir collaborateur confirmé en paie » et « Devenir responsable du pôle social ») organisés chaque année par l'ISFEC.
} 


\section{LE MARCHÉ DE LA FORMATION PROFESSIONNELLE COMME MARCHÉ DE SINGULARITÉS}

Les caractéristiques de la licence en font, selon nous, au moment de sa création et alors que le métier de collaborateur social est émergent, un produit de formation « singulier». Nous sommes en présence d'un produit d'échange entre le futur collaborateur et l'employeur (la formation) donnant lieu à des relations marquées par l'incertitude sur la qualité du produit (Karpik, 2007). Nos observations montrent que la gestion de cette « singularité » a été rendue possible par les effets de multiples formes de proximité. Cependant, des tensions récentes semblent indiquer que la dynamique est marquée par un glissement des formes de proximité et l'arrivée de nouveaux acteurs.

\section{La Licence professionnelle, un dispositif de confiance?}

Lieu d'échange de " services personnalisés 》 (Figure 1), le marché de la formation du collaborateur social prend pour partie les traits d'un marché des singularités. D'une part, la formation peut être relativement ciblée et individualisée grâce à la modularisation et la VAE mais elle est aussi très imbriquée au poste de travail du collaborateur social grâce à l'alternance. Véritable rouage des organisations « apprenantes », la licence assure l'appariement sur le marché du travail du collaborateur social. Elle est encastrée dans une relation de parcours (c'est-à-dire de la sélection à l'entrée en formation jusqu'à la prise de poste en cabinet) où l'IUT et les professionnels sont co-acteurs du recrutement dans la formation puis dans l'emploi.

La singularité tient aussi à l'incertitude sur le contenu exact du poste de collaborateur social. Dans la phase d'émergence du métier, les acteurs rencontrent des

Figure 1

La licence au cœur d'une relation de service

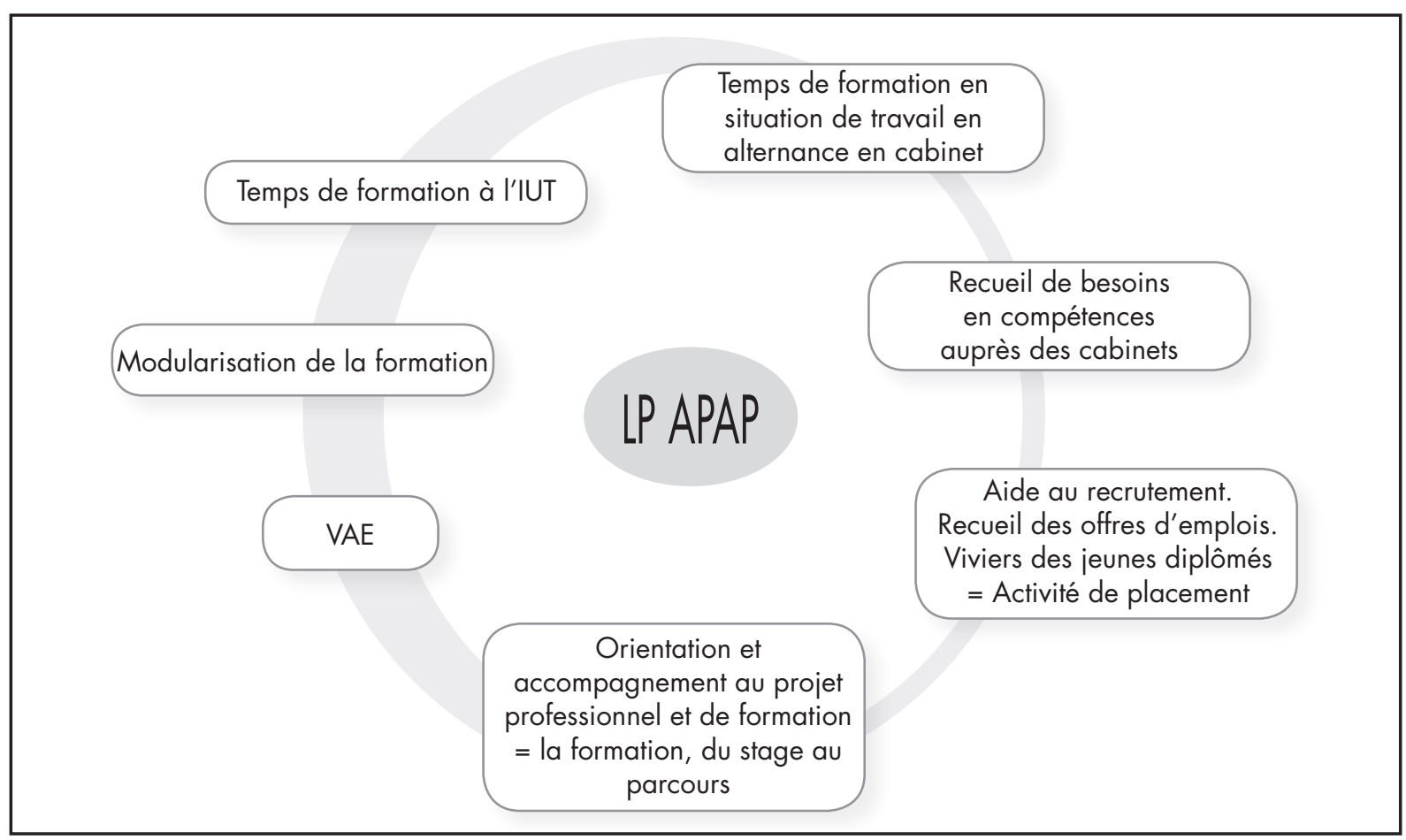

Source : réalisée par l'auteur.

LP APAP : Licence professionnelle « Assistant paie et administration du personnel ». 
difficultés à appréhender les compétences nécessaires, entendu ici la « qualité » du collaborateur, d'autant qu'elles semblent se construire en partie « chemin faisant » et en situation de travail. La qualité n'est pas immédiatement connue par les acteurs. Elle est à construire. Or, selon L.Karpik, dans ce contexte marqué par une forte incertitude sur la qualité des biens échangés, le marché repose sur des dispositifs de coordination qui assurent la confiance et permettent de réaliser un accord sur la qualité du produit. L'incertitude est surmontée par des dispositifs impersonnels ou personnels. Les premiers ne nécessitent pas de connaître personnellement quelqu'un pour acquérir la connaissance nécessaire pour se procurer le bon produit. Ils peuvent reposer sur une connaissance à distance (la consultation d'un catalogue de formation par exemple) ; au contraire, les seconds nécessitent d'établir des relations personnelles pour accéder à l'information. En phase de création, et alors que le marché du travail du collaborateur social est un marché restreint (à l'inverse par exemple de celui de l'assistant RH qui est beaucoup plus étendu), la licence est assez proche d'un dispositif de coordination personnel dans la mesure où la coordination repose sur l'encastrement des professionnels et des enseignants dans un réseau local.

À ce stade, il est intéressant de combiner l'approche par les singularités et l'approche proximiste. Dans l'exemple de la licence, différents dispositifs de coordination - arrangements institutionnels - semblent se succéder. Ils mobilisent chacun différentes formes de proximité. La typologie de la proximité proposée par les chercheurs de "l'école de la proximité » peut donc nous fournir un cadre d'analyse du processus de construction de la licence puis de la dynamique des relations entre acteurs. Cette école s'est développée au début des années 1990. Elle rassemble des géographes et des économistes et étudie la dimension spatiale de la coordination en insistant sur le caractère pluriel de la proximité. La typologie développée par Bouba-Olga et Grossetti (2008) distingue la proximité spatiale et la proximité socio-économique ; cette dernière étant elle-même décomposée en proximité de ressources et proximité de coordination.

La proximité de ressources se fonde sur les caractéristiques individuelles des acteurs (cognitives ou matérielles) indépendamment des possibilités de coor- dination dont ils disposent ; la proximité de coordination quant à elle, renvoie à tout ce qui facilite ou freine la coordination des acteurs (proximité relationnelle ou proximité de médiation). La proximité relationnelle renvoie à la littérature relative à "l'encastrement » selon laquelle les acteurs se coordonnent par les réseaux sociaux. Mais ceux-ci s'appuient aussi sur des ressources de médiation (journaux d'offres d'emploi, cabinet de recrutement, moyens de communication, etc.) qui mettent en contact les acteurs (les employeurs et les salariés sur le marché du travail).

Cette présentation rapide des différentes formes de proximité permet de pointer la mixité des formes de proximité sur laquelle s'appuient les différents acteurs du marché du travail et de la formation du collaborateur social.

Nos observations révèlent en effet que les relations entre les universitaires et les représentants des cabinets se sont d'abord exclusivement déclinées à un niveau local : l'instance ordinale régionale, les cabinets et l'IUT. La coordination repose donc au départ sur une proximité spatiale associée à une proximité de ressource cognitive et matérielle. La proximité spatiale a favorisé l'établissement de relations informelles ou « de confiance ». Les relations entre l'IUT et le CROECB s'appuient sur des relations entre les individus composant ces deux organisations, ce qui peut se lire comme un cas classique «d'encastrement». Le partenariat se noue au départ sur une base « locale », à partir de liens interpersonnels plus ou moins directs : même formation, rencontre dans le cadre de l'Interprofession. Il démarre de manière assez souple, en raison des relations informelles et des contacts fréquents entre les partenaires. Les relations entre l'IUT et les professionnels existent aussi déjà grâce à la participation à des activités d'enseignements en DUT (diplôme universitaire de technologie) et le recrutement de stagiaires de deuxième année. Elles préexistent donc à la création de la licence. Le DUT est une première ressource de médiation. Sous cet angle, la licence ne représente pas une réelle innovation. La notion d'encastrement fonctionne en quelque sorte à l'envers : ce sont les liens individuels qui sont au départ « encastrés » dans le DUT; ils vont s'en détacher ensuite pour créer la licence. La licence permet de mettre en contact les futurs employeurs et collaborateurs qui ne sont pas au préalable en relation. C'est la raison 
pour laquelle elle constitue une nouvelle ressource de médiation. Son émergence relève de la dynamique de relations existantes sur le territoire breton et sur lequel se sont conjugués les intérêts des différents acteurs. Il s'agit donc d'une " ressource du territoire », qui s'est adaptée à des besoins localisés. La singularité est «proxémique » au sens de Marchesnay (2008), c'està-dire qu'elle repose sur des ressources issues de la proximité spatiale, de ressources territoriales offrant une occasion de singularité.

Plusieurs formes de proximité ont été sollicitées dans la construction de la licence. Nous étions dans une situation où la proximité de ressource était faible, c'est-à-dire dans une situation d'inadéquation entre les ressources. L'impératif d'une forte montée en compétence pour le métier de collaborateur social, conjugué à un problème d'attractivité et d'informations sur le métier de collaborateur social, avait créé un éloignement entre les recruteurs et les candidats dans l'espace de ressources (Bouba-Olga et Boudu, 2009). La licence a permis de créer de l'information sur les compétences nécessaires à l'exercice du métier de collaborateur social. Cette information, ressource commune et partagée par l'ensemble des acteurs du réseau, a dès lors généré une « proximité de ressource » qui a permis de réduire l'éloignement et de faciliter l'appariement entre les futurs collaborateurs et les employeurs. Le comité de perfectionnement annuel $^{9}$, le renouvellement du dossier d'habilitation (tous les 4 ans), les visites de stage, les entretiens d'accompagnement des candidats à la VAE ainsi que les jurys VAE constituent des outils de proximité. Leur rôle est de réorganiser l'information mais aussi de créer l'information. Alors que certaines inquiétudes pèsent parfois sur l'efficacité des intermédiaires sur le marché du travail ${ }^{10}$, notre recherche montre ainsi que l'action des intermédiaires engagés dans une démarche de proximité peut devenir vertueuse, et support de l'efficience des marchés. La mise en place

\footnotetext{
9 Le comité de perfectionnement, instance de pilotage de la formation, est constitué de l'ensemble des intervenants et des représentants des différents partenaires (Conseil régional de l'Ordre des experts comptables, Association nationale des directeurs ressources humaines (ANDRH) en Bretagne, Organisme paritaire collecteur agréé (OPCA) AGEFOS PME.

10 Idée selon laquelle les intermédiaires aggraveraient la dissymétrie de la relation entre employeur et candidat à l'emploi en dé-contextualisant les appariements (Bureau et Marchal, 2005).
}

de relations de proximité socio-économique confère tout son potentiel à la proximité spatiale.

\section{La dynamique d'un marché en tensions ou la fin de la singularité}

Six ans après la création de la formation, plusieurs tensions se manifestent. Elles sont liées à l'évolution de certaines règles de fonctionnement du marché du travail et de la formation du collaborateur social. Les travaux de M. Bel (2005) montrent que les relations entreprises-organismes de formation, la nature et le rôle des intermédiaires mobilisés se définissent dans une relation au territoire qui peut varier selon les types de situation. Dans sa définition institutionnaliste du marché du travail, F. Lefresne (2002) nous offre la possibilité de prolonger cette analyse en l'inscrivant dans une perspective dynamique du marché. Selon elle, le marché du travail est "une institution dans laquelle les procédures d'allocation, de mobilité et de rémunération du travail obéissent à un ensemble de règles formelles et informelles stabilisées issues de rapports sociaux » (p. 1248). Or, ces règles ont un ancrage historique et leur stabilité dépend de la stabilité des compromis établis entre les acteurs. Dans le cas de la licence, le jeu d'acteurs s'est progressivement et partiellement modifié. Les motifs d'investissement et le rapport au territoire se sont transformés sous l'effet de forces contradictoires qui laissent entrevoir plusieurs issues distinctes quant à la régulation à venir du marché et le pilotage de la formation : évolution possible de l'activité des cabinets et donc de leur besoin en compétences ; reprise en main par la profession et volonté de contrôler la formation et l'accès au marché du travail ; réduction de l'incertitude sur les compétences requises d'un collaborateur social.

En premier lieu, le processus de professionnalisation reste inachevé et, selon nous, fortement conditionné par les choix stratégiques qu'opèreront les cabinets d'expertise comptable dans les années à venir ${ }^{11}$. Par ailleurs, le métier de collaborateur social commence à être identifié par les activités qu'il recouvre et les

11 Faute d'une rentabilité suffisante et des exigences de leurs clients, certains cabinets commencent à externaliser la paie auprès de prestataires extérieurs pour se concentrer sur le conseil social et $\mathrm{RH}$. 
compétences nécessaires pour l'exercer ; cependant, il lui manque encore une reconnaissance conventionnelle qui se traduirait par une position dans la grille de classification. Reconnaissance de la qualification très attendue par les collaborateurs en poste : « $L a$ convention collective des cabinets d'expertise comptable et commissaires aux comptes ne reconnaît pas encore la fonction "sociale" dans sa classification. Une évolution devrait prochainement intervenir, et l'obtention de la licence professionnelle, par le biais de la VAE, me permettra une certaine reconnaissance dans le cadre d'une future évolution professionnelle » (Candidat VAE).

Cependant, cette reconnaissance tarde à venir.

Selon un processus dynamique d'autonomisation, le partenariat doit en quelque sorte se reconstituer à un niveau national. En se désencastrant du réseau initial localement constitué, il doit s'affranchir des collaborations nouées autour de la licence. À cet égard, une réflexion a été menée au sein de la branche animée par le Conseil supérieur de l'Ordre des experts-comptables. Elle a abouti, en 2010, à la création d'une licence professionnelle « les métiers de la comptabilité $»^{12}$. Elle traduit la volonté de la profession d'avoir « son » diplôme et de piloter le système de formation. Lors de la mise en place du nouveau cursus comptable avec le diplôme de comptabilité et de gestion (DCG) et le diplôme supérieur de comptabilité et de gestion (DSCG), une démarche similaire de normalisation des intitulés et des contenus avait été réalisée avec la transformation des maîtrises de sciences et techniques comptables et financières (MSTCF) en masters « Comptabilité, contrôle, audit » (CCA). Il s'agit cette fois de l'étendre aux licences professionnelles. La procédure d'élaboration de cette licence professionnelle a été conduite sous l'autorité de la profession, sans que les universités n'y soient au départ associées.

L'aboutissement de ce projet revêt deux significations majeures : au niveau de la régulation du marché, il signe le passage à une coordination portée par la profession plutôt que par les établissements et l'Université (Tableau 1) ; au niveau local, la relation au territoire devient une relation instrumentale.

${ }^{12}$ Elle comprend huit spécialités dont la « Gestion de la paie et du social ».
Les professionnels avancent plusieurs arguments pour justifier cette évolution. Le manque de lisibilité dans la carte de formation au niveau national ${ }^{13}$ rendait difficiles d'une part, l'identification et l'étalonnage des compétences professionnelles clefs acquises par les diplômés et d'autre part, la décision de prise en charge financière du coût de la formation par l'AGEFOS-PME ${ }^{14}$. La création d'une LP « Les métiers de la comptabilité » permet de réduire la diversité des appellations et des contenus des maquettes pédagogiques, source de difficulté dans l'évaluation de la qualité des formations.

L'objectif est donc de réduire l'incertitude sur le marché des licences professionnelles préparant aux métiers de la comptabilité ${ }^{15}$. Cela passe par la formalisation et l'uniformisation des formations, indépendamment des «porteurs de projet localisés », et donc une perte en singularité. La licence est conçue comme un cahier des charges que doivent adopter les établissements. Elle se présente comme un « contrat d'adhésion ». En effet, les établissements qui choisissent les maquettes et les intitulés bénéficient des dispenses d'épreuves du DCG, tandis que ceux qui font une offre différente ne peuvent y prétendre. Autrement dit, alors que l'action publique s'était déployée à partir de négociations locales conduites par des porteurs de projets au sein de réseaux locaux, l'action des acteurs privés - ici la branche des expertscomptables et commissaire aux comptes, représentée par son institution ordinale - se déploie à partir de négociations nationales. On s'éloigne de l'hypothèse de M. Bel selon laquelle les licences professionnelles sont un «bien public local». Les acteurs profession-

13 Si plusieurs LP préparant au métier de collaborateur social ont vu le jour en France, depuis 2002, leur intitulé, leur organisation, leur statut pendant la formation, leur recrutement et programmes présentent des différences parfois importantes.

14 L'AGEFOS (Association de gestion de la formation des salariés) PME est l'OPCA de la branche des cabinets d'experts-comptables et commissaires aux comptes.

15 "Les cabinets et leurs clients ont besoin d'une offre de formation simple et lisible pour repérer facilement les compétences des jeunes collaborateurs qu'ils recrutent. Le foisonnement des intitulés et/ou de spécialités des licences professionnelles, la trop grande diversité des contenus ne contribuent pas à donner confiance aux employeurs et nuit à la qualité de l'insertion professionnelle des jeunes diplomés » Extrait d'un document rédigé par un groupe de travail créé à l'initiative de l'OEC et qui décrit les contenus et les objectifs de la LP « Métiers de la comptabilité. Consultable sur le site : www.rhonealpes.experts-comptables.fr/... LicenceProfessionnelle_MétiersComptaGestion_Avril2010.pdf- 
Tableau 1

Typologie des coordinations présidant à l'ouverture des licences professionnelles

\begin{tabular}{|l|c|c|}
\cline { 2 - 3 } \multicolumn{1}{c|}{} & Coordinations portées par les professions & $\begin{array}{c}\text { Coordinations portées par les organismes de } \\
\text { formation professionnelle }\end{array}$ \\
\hline Acteur dominant & Entreprises ou organisations professionnelles & Organisme de formation \\
\hline Logique dominante & Besoins en qualification des entreprises. & $\begin{array}{c}\text { Développement de l'offre de formation : étendre } \\
\text { la carte de formation de l'établissement, attirer des } \\
\text { étudiants. }\end{array}$ \\
\hline Territoire et proximité & $\begin{array}{c}\text { Acceptation instrumentale du territoire. La } \\
\text { formation est répartie sur l'espace national en } \\
\text { fonction des besoins des entreprises. }\end{array}$ & $\begin{array}{c}\text { La proximité géographique a souvent pu favoriser } \\
\text { l'émergence de relations avec les entreprises, mais } \\
\text { ce n'est pas obligatoire. }\end{array}$ \\
\hline $\begin{array}{l}\text { Intermédiaires } \\
\text { Relation entreprises-orga- } \\
\text { nismes de formation }\end{array}$ & $\begin{array}{c}\text { Relations fortes, structurées et relative- } \\
\text { ment stables fondées sur une organisation } \\
\text { professionnelle. }\end{array}$ & $\begin{array}{c}\text { Quelquefois, une organisation professionnelle, mais } \\
\text { le plus souvent cette fonction est assurée par l'orga- } \\
\text { nisme de formation. }\end{array}$ \\
\hline
\end{tabular}

Source : Bel (2005, p. 22).

Note : Seul un extrait du tableau est reproduit ici. La typologie complète inclut deux autres modes de coordination (coordinations portées par la dynamique de développement local et coordinations soutenues par des organismes de formation académiques), non utiles au cas de licence que nous examinons dans notre article.

nels dominent dans cette construction ; et ce, grâce à une organisation collective structurée - l'institution ordinale mais aussi les AGEFOS-PME et à venir l'Observatoire de branche - qui sert d'intermédiaire dans l'étude et la traduction des besoins en compétences à acquérir et dans l'évaluation des besoins quantitatifs. Ce nouvel « arrangement institutionnel » illustre une évolution du rapport qu'entretiennent le territoire et la formation ; de même, il signe la fin de « l'esprit » ou de la singularité des licences professionnelles qui pourraient avoir constitué une étape «d'innovation ». Un mouvement se dessine au sein duquel la proximité spatiale et l'encastrement relationnel demeurent importants (déplacement dans les cabinets lors des placements puis des visites de stage ; comités pédagogiques, jurys VAE, etc.) mais sont instrumentalisés par un encastrement institutionnel au sein d'un réseau étendu et national. À ce jour, l'IUT de Rennes a choisi de ne pas signer ce « contrat d'adhésion ». Il a considéré que la recherche d'équivalences prévues avec le diplôme de comptabilité et de gestion (DCG) n'était pas avérée parmi les étudiants (actuels et anciens). Les enquêtes réalisées révèlent que l'obtention du DCG ne s'inscrit pas dans leur projet professionnel et de formation. Les anciens diplômés ayant repris un cursus de formation après quelques années d'expérience se sont inscrits en master RH ou droit social.

Cette évolution fait aussi écho à une autre tension que nous avons repérée. Des professionnels évoquent une certaine homogénéisation dans les conditions d'exercice des collaborateurs. Ils constatent une tendance à la réduction de l'incertitude initiale sur le contenu du poste et les compétences requises d'un collaborateur social. Méconnu au début des années 2000, le métier est aujourd'hui mieux identifié. Les organismes de formation y voient un marché très porteur. Ils tentent d'imiter le produit « singulier » d'origine, participant à une certaine désingularisation et à la standardisation du produit. L'offre de formation en Bretagne et au niveau national s'est donc développée ; ainsi, le marché devient plus actif et concurrentiel et se rapproche du modèle de régulation marchande (Bel, 2005). Cette situation traduit une transition du marché de la formation vers un mode de régulation au sein duquel les futurs collaborateurs - mais aussi les cabinets - jouissent d'une liberté de choix en vertu d'un marché concurrentiel d'organismes de formation qui rivalisent pour attirer les meilleurs candidats. Les 
acteurs sont plus autonomes et moins en recherche d'un marché organisé et construit pour définir le produit de formation et organiser leur relation dans la durée. Les organismes de formation (dont l'IUT) s'adaptent à la demande et sont soumis à des contraintes de rentabilité. Ils semblent alors plus contraints d'adopter des stratégies qui se rapprochent plus d'une « mise en marché » d'un produit de formation que de l'élaboration d'un projet de formation co-construit avec les partenaires professionnels. Sous cet angle, le marché du travail et de la formation du collaborateur social n'exclut donc pas une dynamique de désingularisation (Karpik, 2007). Cependant, la « standardisation » de la licence reste partielle. Elle est en effet restreinte à certaines activités qui n'exigent pas de proximité dans la relation avec le futur collaborateur et le cabinet d'expertise comptable et de personnalisation de la prestation.

Soulignons aussi que, comme pour de nombreuses licences professionnelles, le rôle du responsable de formation, "entrepreneur de formation » (BEL, 2005), est primordial dans la coordination et l'animation du réseau d'acteurs (enseignants, intervenants professionnels, conseil régional de l'Ordre des experts comptables, administration de l'université, Opca). Les projets de licences professionnelles relèvent, dans de nombreux cas, d'un réseau de relations personnelles d'un enseignant ; dès lors, cela leur confère une fragilité (ces relations sont en effet susceptibles de s'affaiblir ou de disparaître) et hypothèque la solidité du réseau et sa capacité à se maintenir dans la durée : « La singularité est fortement liée à la capacité entrepreneuriale d'un ou plusieurs individus : leur disparition, leur changement de stratégie, leur vieillissement viennent remettre en cause la pérennisation de la singularité » (Marchesnay, 2008, p. 99).

Enfin, on note une évolution des motifs d'investissement des professionnels locaux dans la création de la licence. « Outil de formation » au métier de collaborateur social mais aussi « outil de recrutement» et d'alimentation des emplois, elle a accompagné une stratégie de positionnement de certains cabinets sur le marché très concurrentiel de l'offre de prestations RH. Ces cabinets rencontraient des difficultés à formaliser leur GRH pour développer des marchés internes et fidéliser leurs collaborateurs expérimentés. Mais il semble aujourd'hui que le caractère spécifique de certaines compétences nécessaires à l'exercice en cabinet (la « culture cabinet», la relation client, etc.) n'avait pas été suffisamment perçu par les responsables RH des cabinets. Un expert-comptable très impliqué dans la création de la licence reconnait ainsi que le recrutement externe de jeunes pose aujourd'hui des difficultés liées à un turn-over important. Au sein de son cabinet, les prochains recrutements favoriseront des professionnels plus expérimentés, quel que soit le diplôme mais avec un "profil cabinet expertise comptable »: «Je ne cherche pas forcément un individu qui possède un diplôme en social. Les "profils entreprise" ont beaucoup de difficulté à s'adapter au travail en cabinet, à passer d'une échéance à une autre, au rythme de travail. On est vraiment sur un marché du travail spécifique » (Responsable service social). Cela suppose en interne de créer des outils de GRH et d'activer le marché interne rendu possible par le mouvement de concentration de son cabinet (à l'image de l'ensemble de la profession comptable).

En nous appuyant sur le cas d'une licence professionnelle «Assistant paie et administration du personnel », nous avons cherché à éclairer les motifs et les modalités de coordination, au niveau local, entre acteurs publiques et privés sur le marché du travail et de la formation d'un métier émergent.

La présentation des faits a permis de faire ressortir, dans une première étape, le caractère non standard du produit (la formation), bien singulier. Elle a montré l'intérêt d'un cadre d'analyse qui prenne en considération la co-construction, par l'ensemble des acteurs du marché, de la qualité du produit (la formation). La formation relève de plus en plus d'un service personnalisé qui suppose l'existence de relations de proximité entre les acteurs du marché.

Plusieurs formes de proximité sont intervenues successivement, et parfois conjointement, suggérant une évolution du modèle de coordination. Partant d'une situation où la proximité de ressource était faible, la création de la licence a généré une proximité de ressource ; celle-ci a permis de réduire l'éloignement entre les futurs collaborateurs et les employeurs et de faciliter leur appariement. Proximité socio- 
économique et proximité spatiale on été combinées de manière vertueuse. Dans une seconde étape, la « singularité » de la licence, tenant au caractère émergent du métier de collaborateur social, s'est atténuée. On passe à une coordination portée par la profession plutôt que par les établissements et l'Université. Enfin, au niveau local, la relation au territoire devient une relation plus instrumentale.

La licence professionnelle a été conçue par le ministère de l'Éducation nationale comme une incitation à l'innovation sous condition de partenariat (milieu professionnel, acteurs économiques...) (Maillard D., Veneau P., Grandgérard C., 2004). Si, au niveau local, une grande initiative est laissée aux partenaires, les projets sont régulés au niveau national par la commission d'habilitation. Notre investigation a retracé les conditions de la genèse d'une licence et de son évolution au niveau local sur plusieurs années. Elle témoigne d'une volonté récente de "reprise en main » au niveau national, sous l'impulsion des professionnels.

La dynamique récente du marché confirme aussi l'instabilité propre aux marchés institutionnels, conséquence de la difficulté à organiser, dans le temps, l'action des différents acteurs et leurs relations de proximité. Elle donne raison à Marchesnay lorsqu'il écrit que « en l'espèce, rien n'est donné, une singularité apparemment pérenne peut s'évanouir au gré des évènements » (p. 103).

\section{Bibliographie}

Bel M. (2005), « Conditions de cohérence des politiques de formation dans le contexte français de décentralisation », dans J.L. Guyot, C. Mainguet, B. Van Haeperen (Eds.), La formation professionnelle continue. Enjeux sociétaux, De Boeck, pp. 117-133.

Bel M. (2005), « Politique publique décentralisée : quel rôle pour la proximité ? Le cas de l'ouverture des licences professionnelles ", Economie publique, $\mathrm{n}^{\circ} 16$, pp. 3-26.

Bouba-Olga O., Grosseti M. (2008), " Socioéconomie de proximité ", Revue d'Economie Régionale et Urbaine, $\mathrm{n}^{\circ}$ 3, pp. 1-18.

Bouba-Olga O, Bourdu E. (2009), « La question de l'appariement sur les marchés locaux du travail : une analyse en termes de proximité », Gèmes Journées de la Proximité, 14, 15, 16 octobre 2009, Poitiers.

Bureau M.-C. et Marchal E. (2005), Pluralité des marchés du travail et qualité des intermédiaires, Centre d'Etudes de l'Emploi, Document de travail, $n^{\circ} 48$.
Chassard Y. et Kerbourc'h J.-Y. (2007), « Négociation sur la modernisation du marché du travail : ne pas se tromper d'époque », Droit social, $\mathrm{n}^{\circ} 11$, novembre, pp. 1095-1104.

D’Iribarne A. (2003), « Les stratégies marchandes de la formation », Sciences Humaines, hors-série, $\mathrm{n}^{\circ} 40$, pp. 32-35

Gautié J., Godechot O., Sorignet P.-E. (2005), « Arrangement institutionnel et fonctionnement du marché du travail. Le cas de la chasse de tête ", Sociologie du travail, $\mathrm{n}^{\circ}$ 47, pp. 383- 404.

Karpik L. (2007), L'économie des singularités, NRF, Editions Gallimard.

Lefresne F. (2002), "Vers un renouvellement de l'analyse segmentationniste ", Economies et Sociétés, Série « Economie du travail », AB, n 22, pp. 1241-1267.

Maillard D., Veneau P., Grandgérard C. (2004), « Les licences professionnelles. Quelle acceptation de 
la "professionnalisation" à l'université ", Relief.5, Rapport du Cereq, juin.

Marchesnay M. (2008), « Proximités et singularités des TPE », dans A. Jaouen et O. Torres (dir.) Les très petites entreprises. Un management de proximité, Lavoisier, pp. 81-103.
Moysan-Louazel A. et Merel C. (2008), « Le collaborateur social du cabinet d'expertise comptable », Revue Française de Comptabilité, n 407, pp. 31-34.

Rallet A. et Torre A. (2004), « Proximité et localisation », Economie rurale, $\mathrm{n}^{\circ} 280$, mars-avril, pp. 25-40.

\section{Résumé}

\section{Les marchés du travail et de la formation : entre singularités et proximités Le cas du collaborateur social de cabinet d'expertise comptable et $d^{\prime}$ une licence professionnelle}

\section{Anne Moysan-Louazel}

Le rôle de la proximité dans l'efficacité de l'appariement entre offres et demandes sur les marchés locaux du travail et dans la création des licences professionnelles est connu. On sait aussi que la " singularité » de certains marchés nécessite des dispositifs de coordination particuliers. Nous proposons, dans cet article, de mettre en évidence l'intérêt de cette double perspective théorique pour appréhender la question de la dynamique des dispositifs de coordination sur les marchés du travail et de la formation. À cet effet, nous nous intéressons plus particulièrement à la création et à l'évolution récente de la licence professionnelle "Assistant paie et administration du personnel ». Nous montrons une évolution de la singularité du produit de formation et des formes de proximité auxquelles ont recours les acteurs.

\section{Mots clés}

Marché de la formation professionnelle, création-rénovation de diplôme, licence professionnelle, métier de la fonction financière-comptable

Journal of Economic Literature: J 44, I 29 